\title{
COSMIC BACKGROUND RADIATION ANISOTROPIES IN THE FAR INFRARED
}

\author{
P. de Bernardis, M. De Petris, M. Epifani, M. Gervasi, G. Guarini, and S. Masi \\ Dipartimento di Fisica \\ Università di Roma, Italy
}

The motion of the earth with respect to the distant matter frame produces a spectral distortion in the observed background radiation given by

$$
I_{o b s}\left(v_{o b s}\right)=I(v) \gamma^{3}(1+\beta \cos \theta)^{3} \text {. }
$$

The second-order approximation in $\beta$ gives (de Bernardis et al., 1989)

$$
I_{\text {obs }}(v) \approx I(v)\left[1+(3-\alpha) \beta \cos \theta+(3-\alpha) \frac{\beta^{2}}{2}+\left[3-2 \alpha+\frac{1}{2} \frac{v^{2}}{I(v)} \frac{d^{2} I(v)}{d v^{2}}\right] \beta^{2} \cos ^{2} \theta\right],
$$

where $\alpha \equiv(d \ln I / d \ln v)=(v / I)(d I / d v)$. The first term in $\cos \theta$ of equation (2) is the usual dipole anisotropy, the second constant term is the equivalent of the transverse Doppler effect in special relativity, and the third term is the quadrupole anisotropy. It is interesting to note that the quadrupole term is zero in the Rayleigh-Jeans region of the blackbody spectrum: $Q \approx 1 / 2(3-\alpha)(2-\alpha) \beta^{2} \cos ^{2} \theta$.

It has been argued (Fabbri, 1988) that if the source of the extragalactic background radiation (EBR) is responsible for the motion of the Local Group in the direction of the dipole anisotropy, an additional effect arises in the emitted radiation, which adds to the kinematic one: $\Delta I_{v}=(\beta / 2) I_{v}\left(15-8 \alpha_{v}\right)$.

We assume that the spectrum of the background radiation is given by

$$
I_{\mathrm{v}}=B_{\mathrm{v}}\left(T_{0}\right)+\tau\left(B_{\mathrm{v}}\left(T_{d}\right)-B_{\mathrm{v}}\left(T_{0}\right)\right) \text {, }
$$

where $T_{0}=2.74 \mathrm{~K}$ and $T_{d}=3.55 \mathrm{~K}$ are the cosmic background radiation (CBR) and EBR temperatures, respectively, $\tau_{v} \alpha v^{2}$ is the optical depth, and $B_{v}$ is the Planck function (see Matsumoto et al., 1988).

We have calculated the spectrum of the dipole anisotropy in the following cases: purely kinematic effect on a purely Planckian spectrum $(T=2.74 \mathrm{~K})$; purely kinematic effect on the spectrum given by equation (3); gravitational effect on the spectrum given by equation (3); kinematic effect on the spectrum detected by Gush (1981); and spurious dipole due to galactic contamination. A significant excess in the dipole amplitude is expected with respect to the $2.7 \mathrm{~K}$ blackbody for $v>15 \mathrm{~cm}^{-1}$, where the galactic contamination is still negligible. At lower frequencies $\left(\nu \sim 12 \mathrm{~cm}^{-1}\right)$, a significant decrease in the dipole amplitude is expected in the case of the Gush spectrum. In the case of the quadrupole anisotropy, the dust contamination is substantial: interstellar dust emission must be estimated with $10 \%$ accuracy at $v=10 \mathrm{~cm}^{-1}$ in order to discriminate between the kinematic and the spurious quadrupole with signal-to-noise ratio of $\sim 3$ (de Bernardis et al., 1989).

The calculation of small-scale anisotropies is carried out as follows. We use dust blob model proposed by Bond, Carr, and Hogan (1986) using equation (3) for the total background, a power-law evolution for the correlation length $r_{c}(t)^{\gamma}=a^{p} r_{c}(0)^{\gamma}$, and the absorption 
coefficient of graphite and silicate grains to describe the optical properties of the intergalactic dust (Epifani, Guarini, and Melchiorri, 1989). Under these assumptions the autocorrelation function of the EBR+CBR is related to the spectral shape and to the angular scale, $\theta$, by:

$$
C(\theta) \propto \theta^{\gamma-1}\left[\frac{B_{\mathrm{v}}\left(T_{d}\right)-B_{\mathrm{v}}\left(T_{0}\right)}{I_{\mathrm{v}}}\right] .
$$

In the case $\theta>\theta_{c}(z)$, the intensity fluctuations are given by Poissonian fluctuations in the number of overlapping cells $C(\theta) \alpha \theta^{-1}$. If we suppose that the experimental $\Delta I / I$ is totally due to dust inhomogeneities and we take into account the dependence of the present dust density on $z_{d p}$, we obtain the results shown in Figure 1, where experimental data (squares) are reported together with the theoretical behaviour of the fractional anisotropy for different values of the parameter $p$ and for $r_{c}(0)=5 \mathrm{Mpc}$. The dashed and full lines refer to graphite and silicate grains respectively (Epifani, Guarini, and Melchiorri, 1989). The experimental points have been obtained by integrating the quoted values (all of which refer to differential measurements except point B) over the spectral transmission of the experiment of Melchiorri et al.; a constant value $\sigma / \theta=0.5$ has been assumed. The cases $p=0$ (expected for galaxies confined in pancakes) and $p=3-\gamma$ (appropriate to describe stable bound systems; see Bond et al., 1986) are in disagreement with the experimental data for any reasonable redshift of emission. The case $p>2$ is characteristic of dark matter models, in which structures on scales of $10^{6}-10^{7} \mathrm{M} \odot$ form at $z \approx 25-30$. This scenario is consistent with the experimental data if we take $p>3$.

It is evident that decisive tests on the roughness of cosmic primordial matter may only come from properly designed experiments, with suitable wavelength bandwidths and very small angular scales.

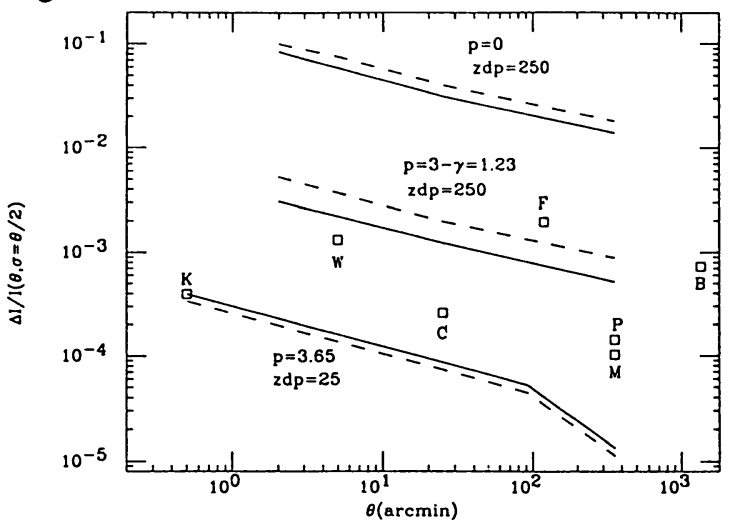

Figure 1. Comparison between observed small-scale anisotropies in the submillimeter region (squares) and theoretical expectations: the dashed and full lines refer to graphite and silicate grains, respectively. Experimental points have been obtained by integrating the quoted values (all of which refer to differential measurements except point B) over the spectral transmission of the experiment of Melchiorri et al. A constant value of $1 / 2$ has been assumed for the ratio $\sigma / \theta$.

\section{REFERENCES}

Bond, J. R., Carr, B. J., and Hogan, C. J. 1986, Ap. J., 306, 428.

de Bernardis, P., et al. 1989, Ap. J. Letters, submitted.

Epifani, M., Guarini, G., and Melchiorri, F. 1989, Ap. J., submitted.

Fabbri, R., 1988, Ap. J., 324, 6.

Gush, H. P., 1981, Phys. Rev. Letters, 47, 745.

Matsumoto, T., et al. 1988, Ap. J., 329, 567. 